\title{
A NOTE ON THE FLOW REGIMES IN THE UNSTEADY, RECTILINEAR FLOW OF A PERFECT GAS
}

\author{
J. A. STEKETEE \\ Dept. of Aeronautics, \\ Delft University of Technology \\ Dept. of Aerospace Engineering \\ University of Michigan
}

\begin{abstract}
Homentropic, unsteady, rectilinear flows can be classified in (i) constant flows, (ii) simple waves, (iii) general flows. After a short discussion of this standard theory, an attempt is discussed to extend this scheme to nonhomentropic flows. The principal result is that a flow with the constraint " $\mathrm{d} u+(1 / \rho a) \mathrm{d} p=0$ everywhere" is physically impossible. In two final sections two suggestions are discussed to remedy the situation, and the linearized case is discussed where analogous features are present.
\end{abstract}

\section{$\S 1$. Equations of motion}

The Eulerian equations of motion for the rectilinear unsteady motion of an ideal gas with constant specific heats can be written in the form

$$
\begin{gathered}
\frac{\partial \rho}{\partial t}+u \frac{\partial \rho}{\partial x}+\rho \frac{\partial u}{\partial x}=0 \\
\frac{\partial u}{\partial t}+u \frac{\partial u}{\partial x}+\frac{1}{\rho} \frac{\partial p}{\partial x}=0 \\
\frac{\partial S}{\partial t}+u \frac{\partial S}{\partial x}=0 \\
p=\rho^{\nu} \exp \left(\frac{S-S_{0}}{C_{\mathrm{v}}}\right) .
\end{gathered}
$$


In these equations $x, t, \rho, u, p$ and $C_{\mathrm{v}}$ have the usual meaning, while $S$ represents the entropy per unit mass. $S_{0}$ is an integration constant, which will be incorporated in $S$ from now on.

The equations (1.1)-(1.4) have been studied extensively, when the flow is homentropic. In that case each fluid element has the same entropy and $S$ reduces to a constant. In the system (1.1)(1.4) the equation (1.3) can then be left out and (1.4) reduces to the form

$$
p=\text { const. } \rho^{\gamma}
$$

It is the purpose of this note to study the different flow regimes that can exist in the more general nonhomentropic case. However, in order to do so, it is expedient to precede the discussion with a concise treatment of the flow regimes in the homentropic case.

\section{$\S 2$. Classification in the homentropic case}

In the homentropic case the equation (1.5) applies instead of (1.4) and (1.3). The adiabatic speed of sound is defined by the well known relation

$$
a^{2}=\frac{\mathrm{d} p}{\mathrm{~d} \rho}=\gamma C \rho^{\gamma-1}=\gamma \frac{p}{\rho} .
$$

Logarithmic differentiation of (2.1) gives

$$
2 \frac{\mathrm{d} a}{a}=(\gamma-1) \frac{\mathrm{d} \rho}{\rho} .
$$

By means of (2.1) and (2.2) $p$ and $\rho$ can be expressed in $a$ in the equations (1.1) and (1.2), so that equations in $u$ and $a$ result. Addition and subtraction of these equations then leads to the system

$$
\begin{aligned}
& \frac{\partial r}{\partial t}+(u+a) \frac{\partial r}{\partial x}=0, \\
& \frac{\partial s}{\partial t}+(u-a) \frac{\partial s}{\partial x}=0,
\end{aligned}
$$

with $r$ and $s$ denoting the Riemann invariants

$$
r=u+\frac{2 a}{\gamma-1}, \quad s=u-\frac{2 a}{\gamma-1},
$$


and

$$
\begin{aligned}
& u+a=\alpha r+(1-\alpha) s, \\
& u-a=(1-\alpha) r+\alpha s,
\end{aligned}
$$

with $\alpha=(\gamma+1) / 4$.

From these equations the principal features of homentropic flows easily follow; through each point $(x, t)$ there pass two characteristics, an $r$-characteristic with a constant value of $r$ and slope $\mathrm{d} x / \mathrm{d} t=u+a$, and an $s$-characteristic with a constant value of $s$ and slope $\mathrm{d} x / \mathrm{d} t=u-a$. Physically, the characteristics represent sound waves, moving with speeds $\pm a$ relative to the fluid.

The different flow regimes, described by the equations (2.3) can be classified as follows:

(i) throughout the flow $r=$ const. and $s=$ const.

(ii) throughout the flow either $r=$ const. or $s=$ const.

(iii) throughout the flow neither $r=$ const. nor $s=$ const. since they differ from one characteristic to another.

It is easily verified that type (i) is the simplest possible flow with $a=$ const. and $u=$ const., hence a uniform steady flow, with two families of straight parallel characteristics. The particle paths, curves with $\mathrm{d} x / \mathrm{d} t=u$, are also straight and parallel.

Flows of type (ii) are the well known simple waves, occurring in a region adjacent to a flow of type (i). If a straight $s$-characteristic forms the boundary between the two regions, the $\gamma$-characteristics from the region with type (i) flow pass into the adjacent region and since the values of $r$ are constant along these characteristics, $r$ is constant throughout the adjacent region. It is then easily verified for a simple wave flow with $r=$ const. that the $s$-characteristics are lines with $u$ and $a$ constant and hence straight (but not parallel) lines. The same applies to the $r$-characteristics in a simple wave flow with $s=$ const. The duties assigned to the two families of characteristics in type (i) flow, that is carrying the same value of $r$ or $s$ and being straight are reduced for the two families in a simple wave flow; one family carries the same value of $r$ (or $s$ ) but drops the attribute of being straight. The other family keeps the attribute of being straight but drops the requirement that each characteristic has the same value of $s$ (or $r$ ).

Considering that a region in the $x, t$-plane is composed of $\infty^{2}$ 
points and that a characteristic is composed of $\infty^{1}$ points, it follows that $u$ and $a$ in a simple wave flow can be described by one, suitably chosen, independent variable rather than two. This also indicates that the flow of type (ii) will display some kind of similarity.

Other characterizations of the simple wave flow appear if the role of the dependent variables $r$ and $s$ and the independent variables $x$ and $t$ are interchanged. A flow of type (i) is mapped in one point of the $r, s$-plane, while the simple wave flow is mapped on a line $r=$ const. or $s=$ const.

The most general flow is type (iii). In that case no simplifications are possible. Both families of characteristics are curved and the mapping of a type (iii) flow in the $h, t$-plane covers an area in the $r, s$-plane.

In what follows, we investigate to what extent similar ideas can be employed to classify the flow regimes described by the system $(1.1)-(1.4)$.

\section{§3. Extended classification in the nonhomentropic case}

The equations (1.1) and (1.2) are first brought in the characteristic form. Logarithmic differentiation of (1.4) leads to

$$
\frac{1}{\rho} \mathrm{d} \rho=\frac{1}{\gamma p} \mathrm{~d} p-\frac{1}{C_{p}} \mathrm{~d} S
$$

and by means of this relation $\rho$ can be eliminated from (1.1). Due to (1.3) equation (1.1) takes the form

$$
\frac{\partial p}{\partial t}+u \frac{\partial p}{\partial x}+\rho a^{2} \frac{\partial u}{\partial x}=0
$$

with a denoting the local adiabatic speed of sound.

Forming a linear combination of (3.2) and (1.2) by multiplying (3.2) with $\lambda_{1}$ and (1.2) with $\lambda_{2}$ the characteristic directions are easily found. They are determined from the requirement that $u$ and $p$ should be differentiated in the same direction. This leads to

$$
\frac{\mathrm{d} x}{\mathrm{~d} t}=\frac{\lambda_{2} u+\lambda_{1} \rho a^{2}}{\lambda_{2}}=\frac{\lambda_{1} u+\lambda_{2}(1 / \rho)}{\lambda_{1}}=K
$$

and to the two values $K_{1,2}=u \pm a$. Clearly, the two characteristic directions are again sound waves travelling upstream and downstream relative to the flow. The characteristic form of the 
system (3.2) and (1.2) is then found to be

$$
\begin{aligned}
& \left(\frac{\partial u}{\partial t}+\frac{1}{\rho a} \frac{\partial p}{\partial t}\right)+(u+a)\left(\frac{\partial u}{\partial x}+\frac{1}{\rho a} \frac{\partial p}{\partial x}\right)=0 \\
& \left(\frac{\partial u}{\partial t}-\frac{1}{\rho a} \frac{\partial p}{\partial t}\right)+(u-a)\left(\frac{\partial u}{\partial x}-\frac{1}{\rho a} \frac{\partial p}{\partial x}\right)=0
\end{aligned}
$$

while (1.3) is in characteristic form.

It is easily found from (1.4), with $S_{0}$ omitted, that

$$
\begin{gathered}
a=\sqrt{\gamma} p^{(\gamma-1) / 2 \gamma} \exp \left(\frac{S}{2 C_{p}}\right), \\
\frac{1}{\rho a} \mathrm{~d} p=\frac{2 \sqrt{\gamma}}{\gamma-1} \exp \left(\frac{S}{2 C_{p}}\right) \mathrm{d}\left(p^{(\gamma-1) / 2 \gamma}\right) .
\end{gathered}
$$

Introducing a function $\Pi$ of $p$ and a function $\Sigma$ of $S$ defined by

$$
\Pi(p)=p^{(\gamma-1) / 2 \gamma}, \quad \Sigma(S)=\sqrt{ } \gamma \exp \left(\frac{S}{2 C_{p}}\right)
$$

the expressions (3.6) can be rewritten

$$
a=\Sigma \Pi, \quad \frac{1}{\rho a} \mathrm{~d} p=\frac{2}{\gamma-1} \Sigma \mathrm{d} \Pi .
$$

The content of (3.4), (3.5) and (1.3) can then be written as follows: in the direction $\frac{\mathrm{d} x}{\mathrm{~d} t}=u+\Sigma \Pi, \quad \mathrm{d} u+\frac{2}{\gamma-1} \Sigma \mathrm{d} \Pi=0$,

in the direction $\frac{\mathrm{d} x}{\mathrm{~d} t}=u-\Sigma I, \mathrm{~d} u-\frac{2}{\gamma-1} \Sigma \mathrm{d} \Pi=0$,

in the direction $\frac{\mathrm{d} x}{\mathrm{~d} t}=u, \quad \mathrm{~d} \Sigma=0$.

The difference with the equations of the homentropic flow is (a) the appearance of the particle paths as a third family of characteristics and (b) the fact that the expressions $\mathrm{d} u \pm 2 /(\gamma-1) \Sigma \mathrm{d} \Pi$ are no longer exact differentials since $\Sigma$ is no longer constant.

We attempt to classify the flow regimes in a similar way as presented in section 2, beginning from the simplest cases. Before doing 
so, it is of interest to notice that the scheme in section 2 was concerned with expressions $r$ and $s$, which did not contain any differentials. In the present scheme only one of the three quantities can be presented in this way: that is we can either say $\mathrm{d} \Sigma=0$ or $\Sigma=$ const., while the expressions $\mathrm{d} u \pm 2 /(\gamma-1) \Sigma \mathrm{d} \Pi$ are in general not exact differentials. With increments $\mathrm{d} \Sigma, \mathrm{d} u, \mathrm{~d} p$ one naturally associates a direction in the $x, t$-plane and we therefore agree that the statement " $\mathrm{d} \Sigma=0$ everywhere" implies that the statement holds for all directions through each point of the domain under consideration.

The simplest flow is obtained if everywhere

$$
\mathrm{d} u+\frac{2}{\gamma-1} \Sigma \mathrm{d} \Pi=0 \quad \mathrm{~d} u-\frac{2}{\gamma-1} \Sigma \mathrm{d} \Pi=0 \quad \mathrm{~d} \Sigma=0 .
$$

Since $\mathrm{d} \Sigma=0$ implies $\Sigma=$ const., the first two expressions in (3.12) are exact differentials. The flow thus obtained is the uniform flow, type (i) of section 2.

There are three types of flow for which two of the three relations in (3.12) are satisfied everywhere, throughout the flow. The flows with $\mathrm{d} \Sigma=0, \mathrm{~d} u+2 /(\gamma-1) \Sigma \mathrm{d} \Pi=0$ and $\mathrm{d} \Sigma=0, \mathrm{~d} u-$ $-2 /(\gamma-1) \Sigma \mathrm{d} \Pi=0$ are homentropic and represent the simple wave flows of type (ii) in section 2. The third type of flow in this class is such that simultaneously $\mathrm{d} u+2 /(\gamma-1) \Sigma \mathrm{d} \Pi=0$ and $\mathrm{d} u-2 /(\gamma-1) \Sigma \mathrm{d} \Pi=0$. It follows that this is a flow with $u=$ $=$ const. and $p=$ const. Since the entropy is not constant, the density will vary. The flow thus obtained occurs in the contact regions which appear in the interaction of shock waves and rarefaction waves in a shock tube. It is clear that the third family of characteristics, the particle paths, consists of parallel straight lines, while the shape of the other characteristics remains unknown as long as $\Sigma$ is not specified.

There are three types of flow in which only one expression of (3.13) is satisfied everywhere.

Consider first the flow for which $\mathrm{d} \Sigma=0$ everywhere. Since $\Sigma=$ $=$ const. in this flow, the flow is homentropic and the first two expressions in (3.12) are exact differentials, so that the Riemann invariants $r$ and $s$ can be written down explicitly. The flow so obtained is type (iii) of section 2 .

The two other flows in this class are the flows in which every- 
where $\mathrm{d} u+2 /(\gamma-1) \Sigma \mathrm{d} \Pi=0$, respectively $\mathrm{d} u-2 /(\gamma-1) \Sigma \mathrm{d} \Pi=$ $=0$ hold. The first one will be discussed in the next section, and the second one, being of the same character, will not be discussed further. Finally, we have the most complicated kind of flow in which none of the expressions in (3.3) is satisfied everywhere. In this type of flow the expressions in (3.3) are satisfied only in the specific characteristic directions as indicated in (3.9)-(3.11).

$\S 4$. The flow with $\mathrm{d} u+2 /(\gamma-1) \Sigma \mathrm{d} I I=0$ everywhere

From the definition it follows that

$$
\mathrm{d} u+\frac{2}{\gamma-1} \Sigma \mathrm{d} \Pi=0
$$

for all directions through all points. Along a characteristic with slope $\mathrm{d} x / \mathrm{d} t=u-\Sigma \Pi$, it follows from (4.1) and (3.10) that $u=$ $=$ const. and $\Pi=$ const. or $p=$ const. Hence the characteristics $\mathrm{d} x / \mathrm{d} t=u-a$ are lines of constant pressure and velocity. Along the characteristics $\mathrm{d} x / \mathrm{d} t=u$ it follows from (4.1) and (3.11) that $u+2 /(\gamma-1) a=$ const. since

$$
\mathrm{d} u+\frac{2}{\gamma-1} \Sigma \mathrm{d} \Pi=\mathrm{d}\left(u+\frac{2}{\gamma-1} \Sigma \Pi\right)-\frac{2}{\gamma-1} \Pi \mathrm{d} \Sigma .
$$

Hence the particle paths are lines with $\Sigma=$ const. and $r=$ const. The particle paths intersecting a characteristic $\mathrm{d} x / \mathrm{d} t=u-a$, do so at the same slope $\mathrm{d} x / \mathrm{d} t$ since $u=$ const. along the characteristics (3.10).

The next point to show is that the flow so obtained contains logical inconsistencies and can therefore not be expected to occur in reality. Consider therefore a region bounded by two particle paths and two characteristics of the family (3.10) (See Fig. 1). Along the latter two characteristics we have $u=u_{1}=$ const., $p=p_{1}=$ const., $I I=\Pi_{1}=$ const., respectively, $u=u_{2}=$ const., $p=p_{2}=$ const., $\Pi=\Pi_{2}=$ const., while along the two particle paths we have $\Sigma=\Sigma_{1}$, respectively $\Sigma=\Sigma_{2}$.

Integrating $\mathrm{d} u+2 /(\gamma-1) \Sigma \mathrm{d} \Pi$ along the particle path $\mathrm{AB}$, we find

$$
u_{2}-u_{1}+\frac{2}{\gamma-1} \Sigma_{1}\left(\Pi_{2}-\Pi_{1}\right)=0
$$




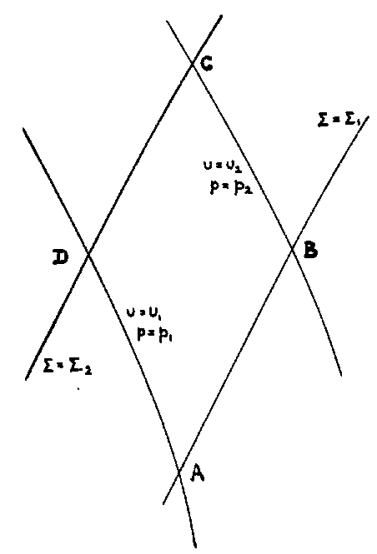

Fig. 1. The $x, t$-plane showing the lines with $\Sigma=$ constant, $u=$ constant, and $p=$ constant.

while along $\mathrm{CD}$

$$
u_{2}-u_{1}+\frac{2}{\gamma-1} \Sigma_{2}\left(\Pi_{2}-\Pi_{1}\right)=0
$$

Since $\Sigma_{1}$ and $\Sigma_{2}$ are different in general we are led to a contradiction. Another way of characterizing the contradiction is to remark that the velocity difference between the points $\mathrm{A}$ and $\mathrm{C}$ depends on the path taken from $A$ to $C$. Taking the path via $A B$, $\mathrm{BC}$ the velocity difference follows from (4.3), taking the path $\mathrm{AD}$, DC one comes to (4.4), with $\Sigma_{2}$ instead of $\Sigma_{1}$.

The contradiction vanishes if $u_{2}=u_{1}, \Pi_{2}=\Pi_{1}$ or if $\Sigma_{1}=\Sigma_{2}$. The same relations should then apply to any other square of characteristics in the flow, and hence we return to the flows with $u=$ const., $\Pi=$ const., $p=$ const., respectively, $\Sigma=$ const. which were already discussed in section 3 .

The only conclusion which remains is that it was incorrect to suppose that a flow can exist in which for all directions through all points the relation (4.1) is satisfied without further conditions or constraints.

\section{§ 5. Discussion}

The failure of the proposed scheme for ordering the nonhomentropic flows indicates that the situation is more complex and subtle than anticipated. 
Two possibilities where modifications can be proposed to remedy the scheme immediately suggest themselves. The first possibility is to admit a flow of the type " $\mathrm{d} u+2 /(\gamma-1) \Sigma \mathrm{d} \Pi=0$ everywhere" only provided the expression is an exact differential. This is the case with the homentropic flow " $\mathrm{d} \Sigma=0$ everywhere" discussed in sections 2 and 3 and is also suggested by a certain analogy with Carathéodory's accessibility theorem, introduced in connection with the second law of thermodynamics $[1,2]$.

Now there are several ways to transform $\mathrm{d} u+2 /(\gamma-1) \Sigma \mathrm{d} I I=0$ into an exact differential. Omitting the homentropic case $\Sigma=$ $=$ const., one can take as the simplest cases $\Sigma=f(\Pi), \Sigma=f(u)$, $\Sigma=f(u, \Pi)$ or $\Sigma=f(u, \Pi, x, t)$.

While it is not difficult to prescribe such a relation, it is an entirely different matter to deduce the necessity of a particular form for $\Sigma$ from the physical situation in a given problem.

In order to show that with the simplest prescriptions of $\Sigma$, the original scheme is not saved yet, consider the case where $\Sigma=$ $=f(u, \Pi)$. Returning to the equations (3.9)-(3.11) for the most general flow, it is clear that the expressions $\mathrm{d} u \pm 2 /(\gamma-1) \Sigma \mathrm{d} \Pi=0$ can be integrated and we deduce that along a characteristic $\mathrm{d} x / \mathrm{d} t=$ $=u+\Sigma \Pi$, an expression $r(u, \Pi)=$ const., while along a characteristic $\mathrm{d} x / \mathrm{d} t=u-\Sigma I I$ an expression $s(u, I I)=$ const. Representing the situation in a $u, \Pi, \Sigma$-space it is clear that the possible values of $u, \Pi$ and $\Sigma$ along an $\gamma$-characteristic are found as the intersection of the surface $\Sigma=f(u, \Pi)$ with the surface $r(u, \Pi)=$ $=$ const. Similar curves are obtained for the possible values of $u$, $\Pi, \Sigma$ along the $s$-characteristics and the particle paths.

Consider now the flow of type "d $u+2 /(\gamma-1) \Sigma \mathrm{d} I I=0$ everywhere". According to the preceding discussion this flow is admissible if the expression is an exact differential and with $\Sigma=$ $=f(u, \Pi)$ we come to the flow $r(u, I)=$ const. The possible combinations of $u, \Pi$ and $\Sigma$ in this flow are represented by the $\infty^{1}$ points composing the intersection curve of the surfaces $\Sigma=f(u, l l)$ and $r(u, \Pi)=$ const. Hence the possible states $u, \Pi, \Sigma$ in this flow, with only one constraint " $\mathrm{d} u+2 /(\gamma-1) \Sigma \mathrm{d} \Pi=0$ everywhere" are as numerous as the states in a homentropic simple wave.

Comparing it with the most general homentropic flow " $\mathrm{d} \Sigma=0$ everywhere", it is easily seen that there $\infty^{2}$ different states are possible; every point of the plane $\Sigma=$ const. gives admissible $u$ 
and $I I$ values. Another way of expressing the same thing is to say that in the most general homentropic flow the Riemann invariants $r=u+2 a /(\gamma-\cdots 1)$ and $s=u-2 a /(\gamma-1)$ can assume all possible values leading to $\infty^{2}$ possibilities.

The reduction in possible states $u, \Pi, \Sigma$ for the flow, "d $u+$ $+2 /(\gamma-1) \Sigma \mathrm{d} \Pi=0$ everywhere" as compared to the flow, " $\mathrm{d} \Sigma=0$ everywhere", is clearly due to the additional function $\Sigma=f(u, I I)$. In the homentropic case the parameter $\Sigma$ has two attributes; in the first place, it is the characteristic parameter for the particle paths, assumed constant for the entire flow in the homentropic case, while secondly it is the simplest assumption to make the two characteristic parameters $\mathrm{d} u \pm 2 /(\gamma-1) \Sigma \mathrm{d} I I=0$ into exact differentials. The function $\Sigma=f(u, \Pi)$ is introduced to make the characteristic parameters $\mathrm{d} u \pm 2 /(\gamma-1) \Sigma \mathrm{d} I I=0$ into exact differentials, but it is not a characteristic parameter assumed constant for the entire flow. The inevitable separation of these attributes in our flow " $\mathrm{d} u+2 /(\gamma-1) \Sigma \mathrm{d} \Pi=0$ everywhere" necessitates the introduction of an extra function and hence imposes more severe constraints on the permissible flows.

It is easily seen that the intersection curve of $\Sigma=f(u, I)$ and $r(u, I T)=$ const. will intersect a plane $\Sigma=$ const. in general in a finite number of points, perhaps even in only one point. This means that the velocity and pressure of a fluid element with a given entropy can only assume a finite number of values $u, \Pi$ independent of $x$ and $t$. Since a fluid element with a different entropy will intersect the curve in another finite set of points, with different values $u, \Pi$ it is in general impossible to maintain continuity in such a flow and we conclude that the flow with $\Sigma=f(u, \Pi)$ and "d $u+2 /(\gamma-1) \Sigma \mathrm{d} \Pi=0$ everywhere" will be in general impossible. One can also check that a flow with $\Sigma=f(u, I T)$ and "d $u+2 /(\gamma-1) \Sigma \mathrm{d} \Pi=0$ everywhere, $\mathrm{d} u-2 /(\gamma-1) \Sigma \mathrm{d} \Pi=0$ everywhere", may be possible with $u=$ const., $\Pi=$ const. and also $\Sigma=$ const., but no longer with an arbitrary entropy distribution as discussed in section 3 .

Whether some of the problems discussed here will vanish, if a more complicated function $\Sigma=f(u, \Pi, x, t)$ is chosen, is a question that will not now be pursued. An example of a flow very similar to the flow discussed here has been studied by Naylor [3] when he assumes that a functional relation of the type $f(u, \Sigma, \Pi)=$ 
$=0$ between the particle velocity and the speed of sound exists.

The second possibility where modifications of the original scheme can be suggested is the following. The original scheme employed the exprestions $\mathrm{d} u \pm 2 /(\gamma-1) \Sigma \mathrm{d} \Pi$ and $\mathrm{d} \Sigma$ to classify the flow regimes. These quantities vanish along the respective families of characteristics, while in the homentropic case they are all exact differentials. These expressions are, however, not the only forms vanishing along the characteristics. Along the characteristics with $\mathrm{d} u+2 /(\gamma-1) \Sigma \mathrm{d} \Pi=0$ also $\mathrm{d} x-(u+\Sigma \Pi) \mathrm{d} t=0$, while, for example, along the particle paths not only $\mathrm{d} \Sigma=0$, but also $\mathrm{d} x$ $-u \mathrm{~d} t=0$. It is possible that a scheme to classify the flows by means of combinations of the two relations, which vanish along each set of characteristics, a more reasonable scheme can be developed. An example, where the same method applied in a similar situation, leads to new results, is the flow studied by Ludford and Martin [4].

\section{§ 6. The linearized case}

It was suggested to the author that the discussion of the preceding pages could be clarified and completed by a concise discussion of the linearized case, where some features of the general nonlinear case are retained.

Beginning with a stationary state of rest $(\partial / \partial t=0, u=0)$ at uniform pressure $\left(p=p_{0}=\right.$ const.) it is clear that the equations (1.1)-(1.3) are identically satisfied, while $\partial \rho / \partial x$ and $\partial S / \partial x$ may possess arbitrary values. Hence the density, entropy and temperature may be assigned as function of $x$ in the stationary state.

Assuming that in the perturbed state

$$
p=p_{0}+p^{\prime}, \quad u=u^{\prime}, \quad S=S_{0}(x)+S^{\prime},
$$

and employing (1.1)-(1.4) in addition to (3.1) one easily finds the linearized equations

$$
\begin{aligned}
& \frac{\partial p^{\prime}}{\partial t}+\rho_{0} a_{0}^{2} \frac{\partial u^{\prime}}{\partial x}=0, \\
& \frac{\partial u^{\prime}}{\partial t}+\frac{1}{\rho_{0}} \frac{\partial p^{\prime}}{\partial x}=0 \\
& \frac{\partial S^{\prime}}{\partial t}+u^{\prime} \frac{\partial S_{0}}{\partial x}=0
\end{aligned}
$$


where the parameters with subscript zero refer to the stationary state and the dashed parameters represent the perturbations. In particular $a_{0}$ is the local speed of sound as given by (3.6).

The physical significance of the equations is clear. The first two are a set of equations for the sound waves (pressure-velocity perturbations). The third equation gives the local change of entropy, with time, expressed as transport, by means of the perturbation velocity, of the stationary entropy distribution. The idea of a characteristic equation, with the particle path as characteristic, associated with (1.3) and (3.11) has vanished in (6.4).

For reasons of symmetry it is elegant to replace the coordinate $x$ by $z$ with $\mathrm{d} x=a_{0} \mathrm{~d} z$. The equations (6.2) and (6.3) then take the form

$$
\frac{\partial p^{\prime}}{\partial t}+\rho_{0} a_{0} \frac{\partial u^{\prime}}{\partial z}=0, \quad \frac{\partial u^{\prime}}{\partial t}+\frac{1}{\rho_{0} a_{0}} \frac{\partial p^{\prime}}{\partial z}=0,
$$

where $\rho_{0} a_{0}$ can be considered as a given function of $z$.

The characteristic form of the equations (6.5) is easily found by the method of section 3. It leads to the result that, along

$$
t-z=\alpha=\text { const. } \quad \mathrm{d} p^{\prime}+\rho_{0} a_{0} \mathrm{~d} u^{\prime}=0,
$$

while along

$$
t+z=\beta=\text { const. } \quad \mathrm{d} p^{\prime}-\rho_{0} a_{0} \mathrm{~d} u^{\prime}=0 .
$$

The difference with the equations (3.9) and (3.10) is that the characteristics are explicitly known in terms of $z$ and $t$ while moreover $\rho_{0} a_{0}$ is a given function of $z$. The expressions $\mathrm{d} p^{\prime} \pm \rho_{0} a_{0} \mathrm{~d} u^{\prime}$ again, are generally not exact differentials.

Classifying the linearized flows along the lines of section 3 , we consider a flow with the condition "everywhere $\mathrm{d} p$ ' $+\rho_{0} a_{0} \mathrm{~d} u^{\prime}=0$ and $\mathrm{d} p^{\prime}-\rho_{0} a_{0} \mathrm{~d} u^{\prime}=0$ ". This is clearly a flow with $p^{\prime}=$ const. and $u^{\prime}=$ const. It is a very elementary flow, where a small constant translation velocity and a small constant pressure are superposed upon the stationary state. Physically speaking this flow is of the same kind as the stationary state.

The next flow to be considered is the analogue of the flow considered in section 4, when only one characteristic quantity, say $\mathrm{d} p^{\prime}+\rho_{0} a_{0} \mathrm{~d} u^{\prime}$ vanishes everywhere. Considering then the characteristics $t+z=$ const., it follows from (6.7) that along these 
characteristics $\mathrm{d} p^{\prime}-\rho_{0} a_{0} \mathrm{~d} u^{\prime}=0$, while also $\mathrm{d} p^{\prime}+\rho_{0} a_{0} \mathrm{~d} u^{\prime}=0$, since the latter expression vanishes everywhere. It follows, that along the characteristics $t+z=\beta=$ const. we have $\mathrm{d} p^{\prime}=\mathrm{d} u^{\prime}=$ $=0$ or $p^{\prime}=$ const., $u^{\prime}=$ const. Introducing the characteristic coordinates $\alpha, \beta$, as defined in (6.6) and (6.7) instead of $z$ and $t$, it follows that in the flow envisaged here $p^{\prime}$ and $u^{\prime}$ will depend on $\beta$ only. Since $2 z=\beta-\alpha$, the requirement " $\mathrm{d} p$ ' $+\rho_{0} a_{0} \mathrm{~d} u$ ' $=0$ everywhere" can then be written

where

$$
P^{\prime}(\beta)+F(\beta-\alpha) U^{\prime}(\beta)=0,
$$

$$
p^{\prime}=P(\beta), \quad u^{\prime}=U(\beta), \quad \rho_{0} a_{0}=F(\beta-\alpha),
$$

and dashes, in (6.8), denote differentiation to $\beta$.

The equation (6.8) can be written as the sum of two terms, one depending on $\beta$, the other depending on $\beta-\alpha$. If the sum of these two terms has to vanish for arbitrary values of $\alpha$ and $\beta$, each term has to be a constant. It follows, that the type of flow envisaged here will only be possible if $\rho_{0} a_{0}=$ const.; with $\rho_{0} a_{0}$ a given function of $z$ the flow will be in general impossible, in agreement with the result found in section 4 .

Physically speaking, the dependence of $p^{\prime}$ and $u^{\prime}$ on $\beta$ only, represents the undistorted propagation along one set of characteristics, and the absence of reflections. In a flow where $\rho_{0} a_{0}$ is a function of $z$, such a propagation without reflections, is therefore shown to be impossible in general.

In order to study the equations (6.6) and (6.7) in more detail it is convenient to employ $\alpha$ and $\beta$ instead of $z$ and $t$ as mentioned before. The equations (6.6) and (6.7) then take the form

$$
\begin{array}{ll}
\text { Along } \alpha=\text { const. } & \frac{\partial p^{\prime}}{\partial \beta}+F(\beta-\alpha) \frac{\partial u^{\prime}}{\partial \beta}=0 \\
\text { along } \beta=\text { const. } & \frac{\partial p^{\prime}}{\partial \alpha}-F(\beta-\alpha) \frac{\partial u^{\prime}}{\partial \alpha}=0 .
\end{array}
$$

Eliminating $p^{\prime}$ and $u^{\prime}$ in turn from (6.10) and (6.11) one is led to equations of Euler-Poisson-Darboux type for $p^{\prime}$ and $u^{\prime}$. This point however will not now be pursued further. 


\section{Acknowledgements}

The author is grateful to professor L. J. F. Broer for the suggestion to complete the discussion by including the linearized case.

The author would also like to express his gratitude to the authorities in charge of the Visiting Netherlands Professorship at The University of Michigan, to his Delft colleagues for taking over some of his duties during the academic year 1970-71 and to his colleagues in Ann Arbor for their hospitality and for providing ideal working conditions.

Received 5 May 1971

In final form 22 February 1972

\section{REFERENCES}

[1] Carathéodory, C., Math. Annalen 67 (1909) 355.

[2] Sneddon, I. N., "Elements of Partial Differential Equations", McGraw-Hill, 1957.

[3] NAylor, D., J. of Math. and Mech. 9 (1960) 1.

[4] Ludford, G. S. S. and M. H. Martin, Comm. Pure Appl. Math. 7 (1954) 45. 